\title{
3D Plant Modelling via Hyperspectral Imaging
}

\author{
Jie Liang \\ Australian National University \\ Australia \\ jie.liangeanu.edu.au
}

\author{
Ali Zia \\ Griffith University \\ Australia \\ ali.ziadgriffithuni.edu.au \\ Xavier Sirault \\ CSIRO \\ Australia \\ xavier.siraultecsiro.au
}

\author{
Jun Zhou \\ Griffith University \\ Australia \\ jun.zhoudgriffith.edu.au
}

\begin{abstract}
Plant phenomics research requires different types of sensors be employed to measure the physical traits of plant surface and to estimate the plant biomass. Of particular interests is the hyperspectral imaging device which captures wavelength indexed band images that characterise material properties of objects under study. In this paper, we introduce a proof of concept research that builds $3 D$ plant model directly from hyperspectral images captured in a controlled lab environment. We show that hyperspectral imaging has shown clear advantages in segmenting plant from its background and is very promising in generating comprehensive $3 D$ plant models.
\end{abstract}

\section{Introduction}

Plant phenomics is an area of plant biology that studies the influence of genetics and environment on both the physical and biochemical traits of plant organisms [7]. One of the main tasks in this area is dissecting plant function and performance via measurement of plant appearance. Such measurements provide inputs to other key tasks in plant phenomics, including investigating carbon partitioning and photosynthesis in plants, as well as finding mechanisms of drought tolerance and flowering behavior. Therefore, robust and accurate measurement plant methods are of great importance.

The development of sensing technology has enabled many measurement tools such as radar, RGB camera, infrared camera and hyperspectral camera be bedded in plant observation process. Among them, of particular interest is the hyperspectral imaging devices, which provide tens or hundreds of contiguous narrow spectral band images indexed by the light wavelength. These band images contain rich information on spectral and spatial distributions of distinct surface materials. They enable more accurate and reliable object detection and material classification than using panchromatic or multispectral imagery. As a consequence, hyperspectral imaging techniques have been widely used in remote sensing, environmental monitoring, and surveillance in agriculture, industry and military [9]. When applied to plant research, hyperspectral imaging has shown success in detecting traits of disease or nutrition deficient [1, 6].

Despite its advantages in object detection and analysis, the research on hyperspectral imaging in computer vision is still very limited. In recent years, thanks to the production of relatively low cost hyperspectral imaging devices, computer vision researchers have started to explore this area. More understanding of the statistical properties of hyperspectral imagery have been reached [4], while some traditional or new computer vision topics have been covered, such as camera sensitivity analysis [10], feature extraction [13], and illumination estimation [8].

In this paper, we address one of the fundamental problems of computer vision, 3D reconstruction, in the context of plant modelling using hyperspectral images. As far as we know, although some research have already incorporated hyperspectral data into 3D models, all these work have not explicitly built 3D models directly from hyperspectral data. For example, Brusco et al presented an interesting work on modeling historical building with multispectral data, while the depth information was captured by a range camera based on laser scanner [2]. Similarly, Nieto et al built 3D model based on depth data captured by a laser scanner and mapped hyperspectral image to 3D Model to display geological mineral information [16]. More recently, Kim et al integrated a hyperspectral camera into a 3D scanning system to enable the measurement of the diffuse spectral reflectance and fluorescence of specimens [12]. 
Our method, on the contrary, attempts to build a 3D model of plant directly from a sequence of hyperspectral images captured in a controlled lab environment. The spectral data is first used to segment plant from its background. Then keypoints are extracted from plants, which are used to find correspondences between a pair of spectral images. Finally a structure from motion based model is developed to reconstruct the 3D plant. The initial results show that the spectral data can be used for effective plant segmentation, which is an important step for 3D modelling. Furthermore, the 3D models produced from difference bands contains mostly consistent structural information of plants, and in some cases, complement each other. This implies that different band images can capture different properties of plant surface. If these models can be properly combined, they will lead to promising approach in building a 3D model that reflects more complete structural information of the plants than that can be reconstructed by traditional systems []. This technique can also be combined with existing 3D plant modelling methods based on laser scanners or Kinect [15] in order to build more accurate plant models.

The rest of paper is organised as follows. Section 2 describes the hyperspectral plant imaging system. Section 3 introduces the proposed 3D plant modelling method. Section 4 presents the experimental results, with conclusions and future work given in Section 5.

\section{Hyperspectral Imaging of Plants}

Our hyperspectral imaging system consists of three main components, i.e. objective lens, a hyperspectral filter, and a high sensitivity camera, with the hyperspectral filter connecting the lens and the camera. In this research, we have used an acousto-optical tunable filter (AOTF) manufactured by Brimrose Cooperation, which supports imaging from $400 \mathrm{~nm}$ to $1000 \mathrm{~nm}$ at about $10 \mathrm{~nm}$ in spectral resolution. A control unit is connected to the filter to let the light in designated wavelength pass through to reach the camera. By scanning through the visible to infrared wavelength, grayscale images can be generated to form different bands of the hyperspectral image. The output of the imaging process is a data cube with the first two dimensions show the spatial positions of pixels, and the third dimension indexes the bands. Therefore, each pixel on the image is a vector of responses across the visible to infrared spectrum.

We collected plant data in a plant phenomics centre. This centre provides integrated plant measurement system that utilises several imaging tools, such as light detection and ranging sensors, thermal infrared cameras, multispectral and RGB cameras to capture high resolution plant data. The imaging lab provides consistent illumination condition to facilitate the imaging process. During the data capture, a plant was put on a turntable platform and transmitted into the workspace. After the plant was positioned, the hyper- spectral camera captured images by scanning through the visible to infrared bands. Then the platform rotated for three degrees to allow another scan being done. This process continued until the plant had been rotated for 360 degrees with all views covered. During the imaging process, camera parameters such as focus length, zoom, exposure time remained unchanged. At last, 120 data cubes were obtained for each plant, covering the whole surface of the plant. Figure 1 shows an plant image example. The first row of the figure shows band images captured at different wavelength from the same angle, while the second row shows images captured at different angles from the same band.

\section{Plant 3D Modeling}

The proposed 3D modelling method contains three steps, which are image quality improvement, plant segmentation, and 3D reconstructing. The first two steps can be considered as the preprocessing steps.

\subsection{Image Preprocessing}

The hyperspectral images often suffer from noise and cross band misalignment. The noises mainly come from the narrow band of light that is allowed to pass the hyperspectral filter within short period of time. Although our camera is highly sensitive, the signal to noise ratio is still low, especially in the short wavelength range where the light intensity is low. To reduce the influence of these bands, those with very low signal to noise ratio were removed from the data. Then the rest band images were smoothed using a Gaussian filter.

Misalignment of band image can be caused by the chromatic abberation of camera lens, or the misalignment of grating component in the tunable filter. Then light in different wavelength follows slightly different transmission path before reaching camera. In order to reduce the misalignment, we calibrated each band image against an anchor band image at $790 \mathrm{~nm}$. This was done by maximising the mutual information of every band to the anchor band, so that the following transformation matrix can be optimised

$$
\left[\begin{array}{l}
x^{\prime} \\
y^{\prime} \\
1
\end{array}\right]=\left[\begin{array}{ccc}
s \cos (\theta) & -s \sin (\theta) & t_{x} \\
s \sin (\theta) & s \cos (\theta) & t_{y} \\
0 & 0 & 1
\end{array}\right]\left[\begin{array}{l}
x \\
y \\
1
\end{array}\right]
$$

Where $\left[\begin{array}{lll}x^{\prime} & y^{\prime} & 1\end{array}\right]^{T}$ and $\left[\begin{array}{lll}x & y & 1\end{array}\right]^{T}$ are pixels before and after transformation, respectively. $\theta, \mathrm{s}$, and $t_{x}, t_{y}$ are in-plane rotation angle, scale, and translation parameters. After the transformation matrices had been obtained for each band, we performed a linear regression fitting of the transformation matrices, so as to make the changes smooth across different bands.

After quality improvement operations, the next step is to segment plant image from its background. In this task, 


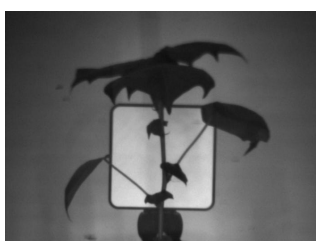

(a)

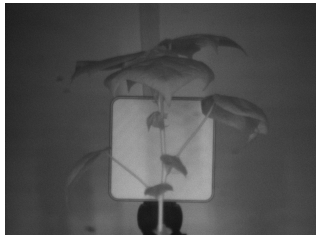

(e)

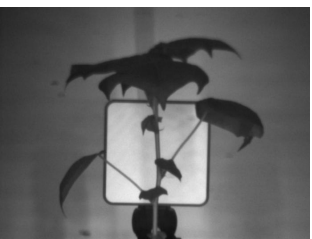

(b)

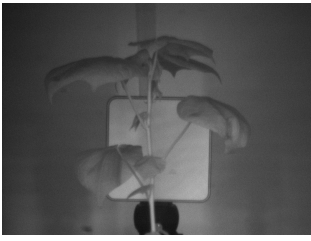

(f)

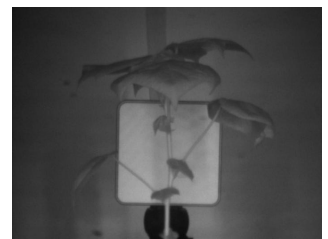

(c)

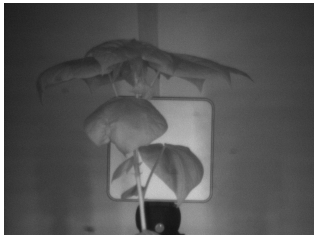

(g)

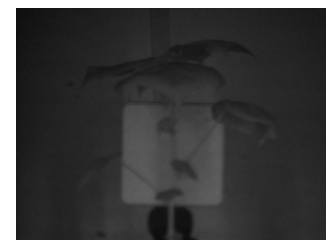

(d)

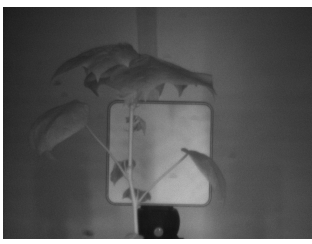

(h)

Figure 1. Hyperspectral images: the first row shows band images captured in $600 \mathrm{~nm}, 700 \mathrm{~nm}, 800 \mathrm{~nm}$ and $900 \mathrm{~nm}$ at 0 degree; the second row shows band images captured in 800nm at 0 degree, 60 degrees, 120 degrees, and 180 degrees, respectively.

hyperspectral data provide much more information on the plant material property of objects than that can be captured from RGB or monochrome images. It shows fine spectral reflectance changes of the plant surface, which is very useful for segmenting plant from its environment. Another spectral property that is very useful for the plant segmentation is that in the near infrared wavelength, plants look much brighter than they appear in the visible range because they have low absorption rate. The hyperspectral data can clearly capture such property, as shown in the last two images of the first row in image 1

To segment plants from its background, we explored two classification methods, K-means clustering and support vector machines. The principle of $\mathrm{K}$-means clustering is to minimize the within cluster sum square error of the whole image, which does not require training data. SVM classifier, on the other hand, tries to find find an optimal hyperplane to distinguish plant pixels from neighboring background pixels.

\subsection{D modeling}

Once the plant is segmented from its background, we build 3D models from the hyperspectral image sequence captured at different angles. Here, we followed a band-byband 3D modelling strategy. At each band, we followed the standard structure from motion method as introduced in [11]. This approach consists of four key steps. They are feature extraction, feature matching, geometry estimation, and $3 \mathrm{D}$ project of key points.

In feature extraction, we employed SIFT keypoints detection and descriptor generation [14]. Then the features in different angle images were matched using the histogrambased descriptors to get the correspondences for combination of images. Because there are 120 images captured from different angles, there would be 7140 pairwise matches.
In terms of 3D reconstruction, suppose the set of image correspondences are $\mathbf{x}_{i} \leftrightarrow \mathbf{x}_{i}^{\prime}$ and it is assumed that these correspondences comes from set of 3D points $\mathbf{X}_{i}$, which are unknown. Similarly, the position, orientation and calibration of the cameras are not known. The reconstruction task is to find the camera matrices $P$ and $P^{\prime}$, as well as the $3 \mathrm{D}$ points $\mathbf{X}_{i}$ such that for each pair of points satisfies

$$
\mathbf{x}_{i}=P \mathbf{X}_{i},
$$

and

$$
\mathbf{x}_{i}^{\prime}=P^{\prime} \mathbf{X}_{i},
$$

There are two steps in the geometry estimation phase. First, fundamental matrix is computed from point correspondences and then the camera matrices are computed from fundamental matrix. To compute fundamental matrix $F$, suppose we have $\mathbf{x}_{i} \leftrightarrow \mathbf{x}_{i}^{\prime}$ correspondences of two images, the fundamental matrix satisfies the condition $\mathbf{x}_{i}^{\prime} F \mathbf{x}_{i}=0$ for all $i$. With the $\mathbf{x}_{i}$ and $\mathbf{x}_{i}^{\prime}$ known, this equation is linear in the (unknown) entries of the matrix $F$. Given at least 8 point correspondences, entries of $F$ can be solved linearly. With more than 8 equations, a least-squares solution is can be adopted. If $P$ and $P^{\prime}$ are pair of camera matrices corresponding to fundamental matrix $F$, then they are computes as follows

$$
P=[I \mid 0]
$$

and

$$
P^{\prime}=\left[\left[e^{\prime}\right] \times F \mid e^{\prime}\right]
$$

where $e^{\prime}$ is the epipole such that $e^{\prime} T F=0$ [11].

The 3D projection of keypoint consists of a process known as triangulation. Let $P$ and $P^{\prime}$ be camera matrices 
and $\mathbf{x}$ and $\mathrm{x}^{\prime}$ be two points in two images that satisfy epipolar constraint $\mathbf{x}^{\prime} T F \mathbf{x}=0$. This constraint may be interpreted geometrically in terms of rays in space corresponding to two image points meaning that $x^{\prime}$ lies on epipolar line $F \mathbf{X}$. So it means that two rays back-projected from image points $x$ and $x^{\prime}$ lie in a common epipolar plane (plane passing through two camera centers). Since two ray lies in a plane they will intersect at some point. This point is $\mathbf{X}$ (3-D point) which is project via two camera point $\mathbf{x}$ and $\mathrm{x}^{\prime}$ in two images [11].

Initially when models were constructed from structured image, problem arose that in order to construct 3D model, SIFT features and matches were required so that correspondence among images could be determined. But unfortunately, the number of SIFT keypoints detected from the plant surface was very few due to the simple structure of plant and low image quality from some noisy image bands. Therefore, there were not sufficient features obtained for each band, and therefore more one disassociated 3D models were generated. Two attempts were tried to alleviate this problem. The first one was to refine edges and structure of images using edge detectors. The second one was to adopt a dense or more versatile model.

Here, we chose to apply canny edge detector [3] on the original image and then performed morphological operation on it. Then the filtered image $F$ was added to the original image $I$ and then be normalized with the maximum value to get the new image which retains structural integrity in an image. This process is shown in following equation

$$
S_{i}=I_{i}+F_{i} / n_{i}
$$

where $i \in\{1, \ldots, n\}$ indexes images captured from different angles for each band).

\section{Experimental Results}

We have carried out experiments on the acquired hyperspectral plant data. Each original data cube consists of 51 bands from $600 \mathrm{~nm}$ to $1000 \mathrm{~nm}$ with $10 \mathrm{~nm}$ interval captured at 120 different angles. Image bands with short wavelength in the visible range were removed because of very low image quality, such that only 41 bands were used for the modelling. The image data were compacted and saved, generating nearly $2 \mathrm{~GB}$ for each plant.

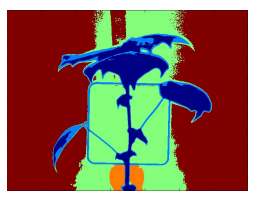

(a)

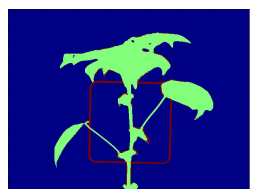

(b)

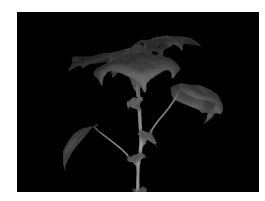

(c)
Figure 2. Segmentation results from a) K-means; b) SVM. c) shows the final segmented plant.
For the segmentation step, we clustered the scene into 4 classes: background, plant, calibration board, and base. The clustering method could not distinguish the boundary of calibration board to the plant itself. Therefore, we also trained an SVM classifier on one hyperspectral image, and applied it to all other images to refine the segmentation. To do so, we used the standard LIBSVM [5] toolbox. Example results are shown in Figure 2, which tells that SVM can generate better segmentation performance than the clustering method. In the feature extraction step, we extracted SIFT descriptors at edge points of plants. Examples of matched keypoints on images from two angles are given in 3 . To generate the 3D model, we adopted virtual-SFM tool [17, 18].

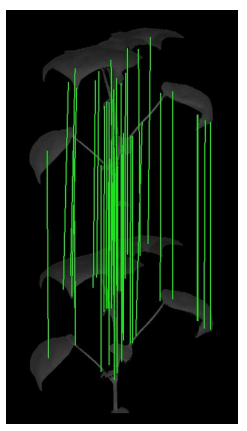

(a)

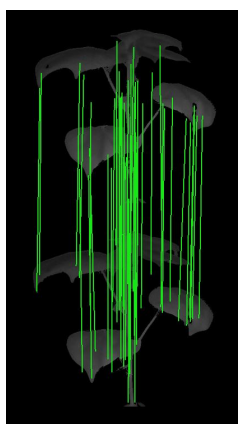

(b)
Figure 3. Examples of matched keypoints.

Finally, some 3D modelling results are displayed in Figure 4. Images in the same row show 3D model observed from different viewing angles when constructed from the same band. From top to bottom, different rows of images show 3D models reconstructed at $600 \mathrm{mn}, 800 \mathrm{mn}, 850 \mathrm{mn}$, and $900 \mathrm{mn}$, respectively. 3D models at $700 \mathrm{mn}$ and $750 \mathrm{~nm}$ can not be reconstructed because the reflectance of plant at these wavelengths is very low.

From the figure, it can be seen that the 3D models obtained are not perfect at this stage. From the 120 images, several models are built. However each model just reconstruct parts of the plant, while the points cloud is not very accurate. This is mainly due to the lack of features in the plant surface. On the other hand, it can be seen that different bands have generated different partial 3D models of the plant. These models compensate to each other, so that we can theoretically build a complete model by merging those partial models.

\section{Conclusions}

The proposed work is the first attempt to build a 3D hyperspectral plant model solely from hyperspectral images. The results show that the spectral information is beneficial to the plant segmentation, and that 3D models reconstructed from different wavelength bands do complement to each 


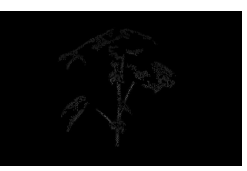

(a)

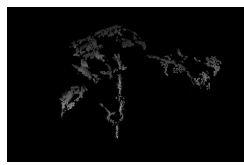

(d)

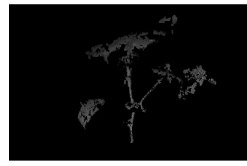

(g)

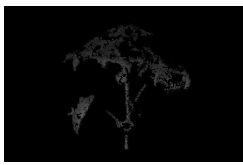

(j)

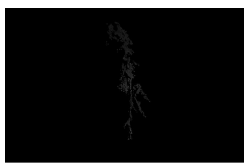

(b)

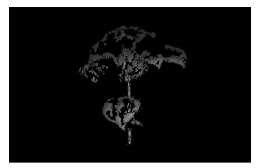

(e)

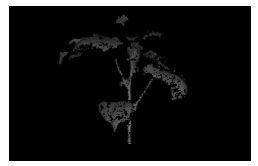

(h)

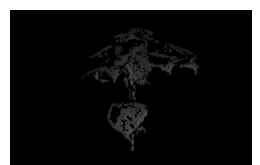

(k)

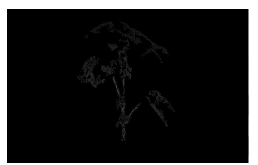

(c)

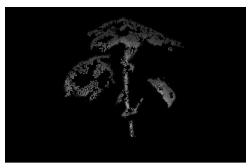

(f)

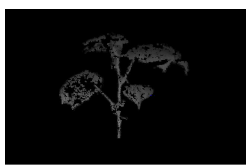

(i)

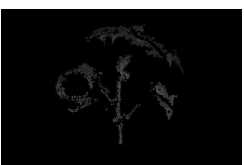

(1)

Figure 4. Different views of the 3D models at different wavelengths.

other. This work opens new dimensions for future work that can be done in 3D modeling from hyperspectral data. One work could be related to developing an effective merging technique for spectral information. Other future research could be to determine new features for spectral data or to improve existing one to suite spectral data needs.

\section{References}

[1] C. H. Bock, G. H. Poole, P. E. Parker, and T. R. Gottwald. Plant disease severity estimated visually, by digital photography and image analysis, and by hyperspectral imaging. Critical Reviews in Plant Sciences, 29(2):59-107, 2010.

[2] N. Brusco, S. Capeleto, M. Fedel, A. Paviotti, L. Poletto, G. Cortelazzo, and G. Tondello. A system for 3D modeling frescoed historical buildings with multispectral texture information. Machine Vision and Applications, 17(6):373-393, 2006.

[3] J. Canny. A computational approach to edge detection. IEEE Trans. Pattern Analysis and Machine Intelligence, 8:679698, 1986.

[4] A. Chakrabarti and T. Zickler. Statistics of real-world hyperspectral images. In Proceedings of the IEEE Conference on Computer Vision and Pattern Recognition, pages 193-200, 2011.

[5] C.-C. Chang and C.-J. Lin. LIBSVM: A library for support vector machines. ACM Transactions on Intelligent Systems and Technology, 2:27:1-27:27, 2011. Software available at http://www.csie.ntu.edu.tw/ cjlin/libsvm
[6] Z. Fu, A. Robles-Kelly, and J. Zhou. Milis: Multiple instance learning with instance selection. IEEE Transactions on Pattern Analysis and Machine Intelligence, 33(5):958977, 2006.

[7] R. T. Furbank and M. Tester. Phenomics technologies to relieve the phenotyping bottleneck. Trends in Plant Science, 16(12):635 - 644, 2011.

[8] L. Gu, A. Robles-Kelly, and J. Zhou. Efficient estimation of reflectance parameters from imaging spectroscopy. IEEE Transactions on Image Processing, 22(9):3548-3663, 2013.

[9] N. Gupta. Hyperspectral imager development at army research laboratory, 2008.

[10] S. Han, Y. Matsushita, I. Sato, T. Okabe, and Y. Sato. Camera spectral sensitivity estimation from a single image under unknown illumination by using fluorescence. In Proceedings of the IEEE Conference on Computer Vision and Pattern Recognition, pages 805-812, 2012.

[11] R. I. Hartley and A. Zisserman. Multiple View Geometry in Computer Vision. Cambridge University Press, ISBN: 0521540518, second edition, 2004.

[12] M. H. Kim, T. A. Harvey, D. S. Kittle, H. Rushmeier, J. Dorsey, R. O. Prum, and D. J. Brady. 3d imaging spectroscopy for measuring hyperspectral patterns on solid objects. ACM Trans. Graph., 31(4):1-11, 2012.

[13] J. Liang, J. Zhou, X. Bai, and Y. Qian. Salient object detection in hyperspectral imagery. In Proceedings of the IEEE International Conference on Image Processing, 2013.

[14] D. G. Lowe. Distinctive image features from scale-invariant keypoints. International Journal of Computer Vision, 60(2):91-110, 2004. 
[15] C. Nguyen, S. Izadi, and D. Lovell. Modeling kinect sensor noise for improved $3 \mathrm{~d}$ reconstruction and tracking. In Proceedings of the Second Joint 3DIM/3DPVT Conference: $3 D$ Imaging, Modeling, Processing, Visualization \& Transmission, pages 524-530, 2012.

[16] J. Nieto, S. Monteiro, and D. Viejo. 3D geological modelling using laser and hyperspectral data. In 2010 IEEE International Geoscience and Remote Sensing Symposium, pages 4568-4571, 2010.

[17] C. Wu. Towards linear-time incremental structure from motion. In 3DTV-Conference, 2013 International Conference on, pages 127-134, 2013.

[18] C. Wu, S. Agarwal, B. Curless, and S. Seitz. Multicore bundle adjustment. In IEEE Conference on Computer Vision and Pattern Recognition, pages 3057-3064, 2011. 